\title{
High-Flow Nasal Cannula Oxygen Delivery during Bronchoalveolar Lavage: A Question of Methodology Influence?
}

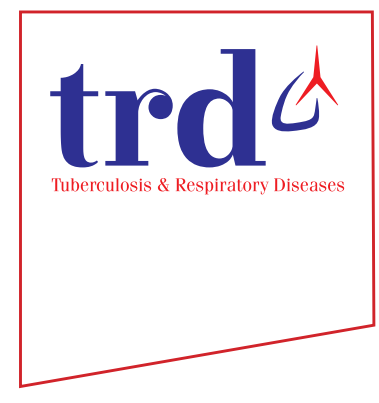

\author{
Subrata Kumar Singha, M.D., P.D.C.C. ${ }^{1}$ (i) and Antonio M Esquinas ${ }^{2}$ (i) \\ ${ }^{1}$ Department of Anaesthesia \& Critical Care, All India Institute of Medical Sciences, Raipur, India, ${ }^{2}$ Intensive Care Unit, Hospital \\ Morales Meseguer, Murcia, Spain
}

Desaturation during bronchoalveolar lavage (BAL) is a critical condition that could delay the procedure and recommendations about the appropriate complementary oxygenation technique are controversial. Recently, high flow nasal cannula (HFNC) is an appropriate alternative to avoid these complications; however, there is scarce information about the real clinical impact ${ }^{1,2}$. In this line we have read with great interest the study by Kim et al. ${ }^{3}$ which described the use of HFNC in patients undergoing BAL. After careful analysis we consider that there are some key aspects that have to be taken into consideration for a proper clinical extrapolation such as firstly, the authors, have used variable flow $(\mathrm{L} / \mathrm{min})$ in the study, which could interfere with the real evaluation of hypoxemic conditions and there is no clear explanation about the same. Secondly, although the authors did not describe significant variations in oxygen saturation levels before, during, and after BAL measured by pulse oximeter (saturation of partial pressure oxygen) values as $94.8 \pm 2.9 \%, 94.6 \pm 3.5 \%$, and $95.2 \pm 2.8 \%$, respectively, it did not exclude the underlying clinical hypoxemic conditions as they may have been maintained by increasing the fraction of the inspired air concentration values. Thirdly, the authors included only the first 24-hour observation period but we are of the opinion that it would be

Address for correspondence: Subrata Kumar Singha, M.D., P.D.C.C.

Department of Anaesthesia \& Critical Care, All India Institute of Medical

Sciences, Tatibandh, Raipur 492099, CG, India

Phone: 91-8518881767

E-mail: subratsingh@gmail.com

Received: Jul. 10, 2018

Revised: Aug. 10, 2018

Accepted: Sep. 6, 2018

(c) It is identical to the Creative Commons Attribution Non-Commercial License (http://creativecommons.org/licenses/by-nc/4.0/). intriguing to extend the observation period for a longer period to properly evaluate late complication if any ${ }^{4}$, which the other clinical studies have described. Last but not the least, we also opine that the protocol for sedation would also influence the desaturation rate as mentioned in a study by Douglas et al. ${ }^{5}$.

We have to exclude positive end expiratory pressure effect during BAL since it would be difficult to interpret the same during bronchoscopy.

\section{Authors' Contributions}

Conceptualization: Singha SK, Esquinas AM. Writing original draft preparation: Singha SK, Esquinas AM. Writing review and editing: Singha SK, Esquinas AM. Approval of final manuscript: all authors.

\section{Conflicts of Interest}

No potential conflict of interest relevant to this article was reported.

\section{References}

1. Miyagi K, Haranaga S, Higa F, Tateyama M, Fujita J. Implementation of bronchoalveolar lavage using a high-flow nasal cannula in five cases of acute respiratory failure. Respir Investig 2014;52:310-4.

2. Messika J, Ben Ahmed K, Gaudry S, Miguel-Montanes R, Rafat C, Sztrymf B, et al. Use of high-flow nasal cannula oxygen therapy in subjects with ARDS: a 1-year observational study. Respir Care 2015;60:162-9.

3. Kim EJ, Jung CY, Kim KC. Effectiveness and safety of highflow nasal cannula oxygen delivery during bronchoalveolar lavage in acute respiratory failure patients. Tuberc Respir Dis 2018;81:319-29. 
4. Simon M, Braune S, Frings D, Wiontzek AK, Klose H, Kluge S. High-flow nasal cannula oxygen versus non-invasive ventilation in patients with acute hypoxaemic respiratory failure undergoing flexible bronchoscopy: a prospective randomised trial. Crit Care 2014;18:712.
5. Douglas N, Ng I, Nazeem F, Lee K, Mezzavia P, Krieser R, et al. A randomised controlled trial comparing high-flow nasal oxygen with standard management for conscious sedation during bronchoscopy. Anaesthesia 2018;73:169-76. 